\title{
Interdisziplinäre Zusammenarbeit in der Rehabilitation
}

\author{
Interdisziplinäre Zusammenarbeit gilt als Grundvoraussetzung für eine zielorien- \\ tierte Rehabilitation. Dieser Gedanke bildet den Schwerpunkt der Tätigkeit der \\ SAR Swiss Association of Rehabilitation, die sich für die medizinischen, sozialen, \\ pädagogischen, beruflichen und gesetzlichen Belange der Rehabilitation einsetzt.
}

\section{Patrick Konietznya}

Hans Peter Gmünder ${ }^{b}$

a Vorstandsmitglied SAR Swiss Association of Rehabilitation

b Präsident SAR Swiss Association of Rehabilitation
Korrespondenz:

of Rehabilitation

Stadthof

CH-6210 Sursee

Tel. 0419260766

Fax 0419260799

patrick.konietzny[at]

klinik-adelheid.ch
SAR Swiss Association

Bahnhofstrasse 7 B

\section{Grundlagen der Rehabilitationsmedizin}

Die Rehabilitation ist ein wichtiger Bestandteil der heutigen Medizin, wobei der Begriff der Rehabilitation national wie auch international unterschiedlichen Konzepten, Strukturen und Massnahmen zugeordnet wird. Die World Health Organization beschreibt Rehabilitation auf ihrer aktuellen Website unter «Health Topics» folgendermassen: "Rehabilitation of people with disabilities is a process aimed at enabling them to reach and maintain their optimal physical, sensory, intellectual, psychological and social function levels. Rehabilitation provides disabled people with the tools they need to attain independence and self-determination» [1].

Die Rehabilitationsmedizin basiert dabei auf dem Modell der funktionalen Gesundheit ICF (International Classification of Functioning, Disability and Health, ICF - Abb. 1), das sich in Verbindung mit einem Gesundheitsproblem an einer ganzheitlichen funktionalen Sichtweise orientiert. Aufgrund dieser mehrdimensionalen Betrachtung, welche die subjektive Sicht des betroffenen Menschen mit einbezieht, ist das Konstrukt der ICF geradezu ideal für die strukturierte Problemanalyse und Zielformulierung in der Rehabilitation. Ziele sind unter anderem, Schädigungen der Körperfunktionen und -strukturen soweit möglich rückgängig zu machen - dies

Abbildung 1

Modell der funktionalen Gesundheit gemäss ICF-Kriterien (WHO 2002) [3]

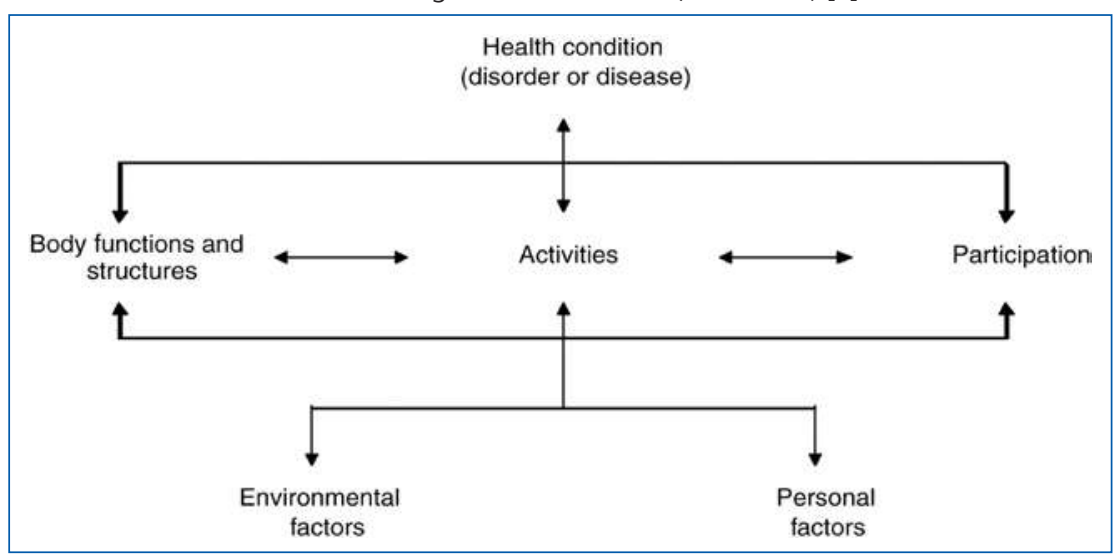

aber immer im Hinblick auf die Erreichung einer bestmöglichen Partizipation unter Einbezug von Kontextfaktoren (personenbezogene Faktoren und Umweltfaktoren) und Anpassung von Hilfsmitteln [2].

\section{Geschichte und Wirken der SAR}

Vor diesem Hintergrund wird deutlich, dass in der Rehabilitation eine der wichtigsten Voraussetzungen zur Zielerreichung die interdisziplinäre Zusammenarbeit ist. Erst das koordinierte Zusammenwirken verschiedener Disziplinen mit ihren Massnahmen (medizinisch, therapeutisch, sozial, technisch und pädagogisch) unter Einbezug von Patienten und Angehörigen sowie der persönlichen Situation im Alltag, Beruf und Freizeit macht eine zielorientierte Rehabilitation möglich. Die interdisziplinäre Zusammenarbeit ist dabei auch gleichzeitig eine der grössten Herausforderungen der Rehabilitation. Dies gilt sowohl für die rehabilitative als auch für die standespolitische Zusammenarbeit. Die SAR möchte hierzu ihren Beitrag leisten und wirkt unter anderem darauf hin, dass interdisziplinäre Zusammenarbeit als Grundvoraussetzung der Rehabilitation anerkannt wird.

Der Grundgedanke der interdisziplinären $\mathrm{Zu}$ sammenarbeit ist noch jung in der Geschichte der SAR. Die Ursprünge der SAR reichen zurück in das Jahr 1960, als die «Kommission für Rehabilitation der Schweizerischen Gesellschaft für Physikalische Medizin» gegründet wurde. Schon von Beginn an arbeiteten Vertreter zahlreicher medizinischer Fachgesellschaften in der Kommission mit. Bereits an der ersten Kommissionssitzung wurde deshalb die Abkoppelung von der Schweizerischen Gesellschaft für Physikalische Medizin beschlossen. Erster Präsident der Kommission war Dr. W. Belart aus Zürich. Viele prominente Mitarbeiter prägten die weitere Entwicklung. 1967 wurde das Präsidium an Dr. W.M. Zinn (Bad Ragaz) übergeben. Es folgte die Namensänderung in «Schweizerische Arbeitsgemeinschaft für Rehabilitation SAR». Gleichzeitig wurde auch der auf 30 Mitglieder festgelegte Numerus clausus fallengelassen und es wurden Vertreter aller medizinischen Fachgesellschaften als Verbindungsleute und Refe- 


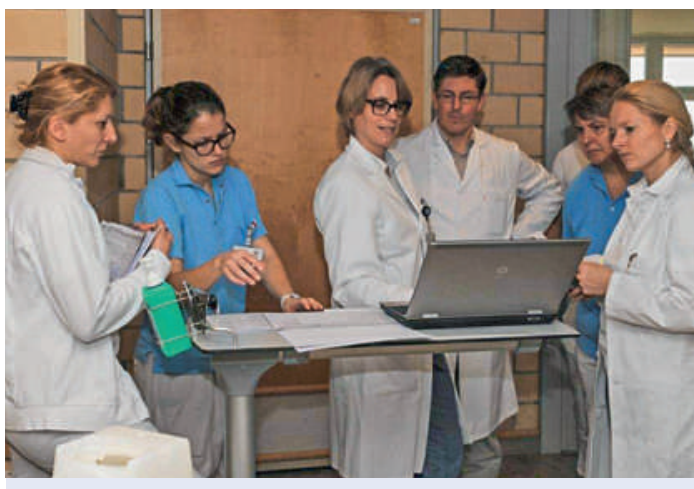

Interdisziplinäre Zusammenarbeit ist entscheidend für eine erfolgreiche Rehabilitation.

renten in die SAR einbezogen. Eine grundlegende und zukunftsweisende Neuerung erfolgte 1983. Die bisher rein ärztliche Arbeitsgemeinschaft wurde zu einem interdisziplinären Gremium, das grundsätzlich allen Rehabilitationsberufen offensteht. Damit wurde die für die Rehabilitation ausschlaggebende Arbeitsweise im multidisziplinären Team in die Praxis umgesetzt. Die SAR blieb auch nicht vor Krisen verschont. Die Beteiligung an den Anlässen der Arbeitsgemeinschaft ging derart stark zurück, dass 1986 ein Antrag zur Auflösung gestellt wurde. Es war in erster Linie der Verdienst des engagierten und überzeugenden Einsatzes des damaligen Präsidenten Profes-

\section{Die SAR wirkt darauf hin, dass interdisziplinäre Zusammenarbeit als Grundvoraussetzung der Rehabilitation anerkannt wird.}

sor Gerber, dass die Arbeitsgemeinschaft überlebte. Unter der Führung von Niklaus Gerber wurde die interdisziplinäre Zusammenarbeit tatkräftig gefördert und die Veranstaltungen wurden auf diese Teamzusammenarbeit ausgerichtet. Erst durch diesen Teamansatz wurde es in der Rehabilitationsmedizin möglich, die verschiedenen Dimensionen von gesundheitlicher Integrität und Behinderung strukturiert und systematisch zu berücksichtigen und in gemeinsam abgestimmten Behandlungskonzepten umzusetzen. Das führte rasch zu einer deutlichen Steigerung des Interesses an der SAR, und die Teilnehmerzahlen an den Veranstaltungen stiegen eindrücklich an. Aufgrund steigender Mitgliederzahlen auch aus der Westschweiz entschied man sich 2012 im Sinne eines einheitlichen Erscheinungsbildes für ein neues Logo und eine erneute Namensänderung in Swiss Association of Rehabilitation. Bis heute umfasst die SAR (als Verein im Sinne von Art. 60 ff. ZGB.) 238 Direktmitglieder und 8 Interessengemeinschaften mit insgesamt
711 Mitgliedern. Das Mitgliederspektrum spiegelt den multidisziplinären Gedanken wider und beinhaltet ärztliche Mitglieder, Delegierte von medizinischen Fachgesellschaften, Verbände medizinischer und sozialer Berufe, private und staatliche RehabilitationsInstitutionen, Organisation der Behinderten- und Selbsthilfe sowie weitere Fachleute des Rehabilitationswesens. Die SAR sieht sich als Ansprechpartner für Fragen der Rehabilitation und handelt selbstbestimmt, interdisziplinär, zukunftsorientiert und ganzheitlich, wobei sie keine Standesinteressen vertritt.

Neben der Multidisziplinarität bestimmen auch andere Themen das Wirken der SAR. So tritt die SAR ein für die medizinischen, sozialen, pädagogischen, beruflichen und gesetzlichen Belange der Rehabilitation in Zusammenarbeit mit Fachgesellschaften, Kostenträgern und Gesetzgebern. Sie fördert wissenschaftliche Arbeiten zum Thema der Rehabilitation aber auch die Rehabilitation in der Aus-, Weiter- und Fortbildung aller Fachdisziplinen. Beispielhaft zeigt sich dies in der Arbeitsgruppe ICF-Plattform der SAR, welche das Wissen betreffend ICF-Anwendungen in Rehabilitationen, das Networking zwischen Health Professionals betreffend ICF-Anwendungen, die Initialisierung von ICF-basierten Instrumenten und die Zusammenarbeit zwischen ICF-orientierter Forschung und Praxis fördern will. So sind die jährlichen ICF-Anwendertagungen mittlerweile ein fester und beliebter Bestandteil zahlreicher Aktivitäten dieser Arbeitsgruppe geworden.

Neben weiteren Bildungsangeboten der SAR bietet das jährlich stattfindende SAR-Forum Platz für ein breites thematisches Spektrum, welches das weite Interesse der SAR und die gute Zusammenarbeit mit anderen medizinischen Fachgesellschaften belegt.

Das mehrmals jährlich erscheinende Informationsbulletin sowie weitere Medienmitteilungen informieren über die Aktivitäten in der SAR, ihrer Partner und zu Themen der Rehabilitation.

Vor dem Hintergrund wachsender Aufgaben im Rehabilitationswesen (Tarifstruktur, ANQ u.a.) wird sich die SAR auch künftig für die Anliegen der Rehabilitation einsetzen und als Ansprechpartner für Fragen der Rehabilitation zur Verfügung stehen. Dabei kommt insbesondere den ärztlichen Mitgliedern als eines der wichtigsten Bindeglieder der interdisziplinären Zusammenarbeit eine besondere Bedeutung $\mathrm{zu}$, weswegen die SAR weiterhin auf einen steten $\mathrm{Zu}$ wachs ihrer ärztlichen Mitglieder hofft.

\section{Literatur}

1 www.who.int/topics/rehabilitation/en.

2 DefREHA $^{\circ}$ - Stationäre Rehabilitation: Spezialbereiche und Definitionen, Ein- und Ausschlusskriterien für die unterschiedlichen Rehabilitationsarten, Version 1.0. H+ Die Spitäler der Schweiz. S. 6-7.

3 ICF, The International Classification of Functioning. Disability and Health. WHO 2002; S. 9. 